\title{
A self-administered, artificial intelligence (Al) platform for cognitive assessment in multiple sclerosis (MS)
}

Seyed-Mahdi Khaligh-Razavi ${ }^{1,2^{*}}$ D, Maryam Sadeghi ${ }^{3}$, Mahdiyeh Khanbagi ${ }^{2}$, Chris Kalafatis ${ }^{1,4,5}$ and Seyed Massood Nabavi ${ }^{2}$

\begin{abstract}
Background: Cognitive impairment is common in patients with multiple sclerosis (MS). Accurate and repeatable measures of cognition have the potential to be used as markers of disease activity.

Methods: We developed a 5-min computerized test to measure cognitive dysfunction in patients with MS. The proposed test - named the Integrated Cognitive Assessment (ICA) - is self-administered and languageindependent. Ninety-one MS patients and 83 healthy controls $(\mathrm{HC})$ took part in Substudy 1, in which each participant took the ICA test and the Brief International Cognitive Assessment for MS (BICAMS). We assessed ICA's test-retest reliability, its correlation with BICAMS, its sensitivity to discriminate patients with MS from the HC group, and its accuracy in detecting cognitive dysfunction. In Substudy 2, we recruited 48 MS patients, 38 of which had received an 8-week physical and cognitive rehabilitation programme and 10 MS patients who did not. We examined the association between the level of serum neurofilament light (NfL) in these patients and their ICA scores and Symbol Digit Modalities Test (SDMT) scores pre- and post-rehabilitation.

Results: The ICA demonstrated excellent test-retest reliability $(r=0.94)$, with no learning bias, and showed a high level of convergent validity with BICAMS. The ICA was sensitive in discriminating the MS patients from the HC group, and demonstrated high accuracy (AUC $=95 \%$ ) in discriminating cognitively normal from cognitively impaired participants. Additionally, we found a strong association $(r=-0.79)$ between ICA score and the level of NfL in MS patients before and after rehabilitation.

Conclusions: The ICA has the potential to be used as a digital marker of cognitive impairment and to monitor response to therapeutic interventions. In comparison to standard cognitive tools for MS, the ICA is shorter in duration, does not show a learning bias, and is independent of language.
\end{abstract}

Keywords: Multiple sclerosis, BICAMS, Digital biomarkers, Integrated cognitive assessment (ICA), Languageindependent, Artificial intelligence (Al)

\footnotetext{
* Correspondence: seyed@cognetivity.com

${ }^{1}$ Cognetivity Ltd, London, UK

${ }^{2}$ Department of Brain and Cognitive Sciences, Cell Science Research Center, Royan Institute for Stem Cell Biology and Technology, ACECR, Tehran, Iran

Full list of author information is available at the end of the article
}

(c) The Author(s). 2020 Open Access This article is licensed under a Creative Commons Attribution 4.0 International License, which permits use, sharing, adaptation, distribution and reproduction in any medium or format, as long as you give appropriate credit to the original author(s) and the source, provide a link to the Creative Commons licence, and indicate if changes were made. The images or other third party material in this article are included in the article's Creative Commons licence, unless indicated otherwise in a credit line to the material. If material is not included in the article's Creative Commons licence and your intended use is not permitted by statutory regulation or exceeds the permitted use, you will need to obtain permission directly from the copyright holder. To view a copy of this licence, visit http://creativecommons.org/licenses/by/4.0/ The Creative Commons Public Domain Dedication waiver (http://creativecommons.org/publicdomain/zero/1.0/) applies to the data made available in this article, unless otherwise stated in a credit line to the data. 


\section{Background}

Multiple sclerosis (MS) is characterized by widespread demyelination and neurodegeneration in the central nervous system [1]. Therefore, cognitive dysfunction is common in MS patients (40-70\% of these patients are reported to have cognitive impairment [2]), and is associated with a higher risk of disease progression in the subsequent years [2]. Cognitive impairment can have significant negative impacts on several domains of daily living activities, such as social functioning, employment [3] and driving [4]. Despite the prevalence of cognitive impairment and its negative impact on patients' lives, cognitive assessment is not routinely carried out for MS patients [5].

Early detection of cognitive impairment in MS could be helpful in the identification of patients at high risk of disability progression and poor clinical outcome [6]. Furthermore, cognition has the potential to be used as a marker of disease progression or treatment efficacy in MS [7, 8]. When patients report a cognitive problem, they are describing a change in function from a previous level; however, the majority of cognitive tests, due to a learning bias $[9,10]$, cannot be used for frequent monitoring of cognitive performance. On the other hand, neuroimaging and fluid biomarkers of disease activity [11-13], while more accurate, are less suitable for frequent monitoring of disease progression and more difficult to integrate into routine clinical practice. Here, we propose an AI-assisted digital biomarker of cognitive function, appropriate for monitoring disease activity.

There is evidence that the afferent visual system is highly vulnerable to MS [14]. Furthermore, deficit in information processing speed (IPS) is the most prevalent cognitive impairment in MS, and can affect the speed of sensory, motor and cognitive processes [15]. We designed an iPad-based rapid visual categorization task [16-18] the Integrated Cognitive Assessment (ICA), that primarily assesses IPS in visuomotor domains. The task is designed to give a sensitive, repeatable measure of IPS, and is additionally shown to be correlated with other cognitive domains, such as verbal memory and visuospatial abilities [19]. The test is software-based, selfadministered and is shown to have little dependency on participants' language, and is not confounded by participants' varying levels of education [19].

To measure the efficacy of the proposed ICA test in detecting cognitive impairment in MS patients, we compared the ICA with the Brief International Cognitive Assessment for MS (BICAMS) [20, 21]. BICAMS is a penand-paper based cognitive assessment battery for detecting cognitive dysfunction in MS patients. The BICAMS battery includes tests of mental processing speed as well as visuospatial and verbal learning, and takes about 15 to 20 min to administer and score.
To further assess the validity of the ICA test as a potential digital biomarker of MS disease activity, we compared ICA test results with participants' level of serum neurofilament light chain (NfL). NfL has been shown to be a valuable fluid biomarker of MS disease activity and treatment response [22], and is associated with clinical and MRI-related measures of disease activity and neuroaxonal damage [23]. Changes in NfL are shown to be associated with changes in global cognition and attention [24]. Furthermore, elevated baseline plasma NfL is a prognostic marker of cognitive decline and neuroimaging measures of neurodegeneration, and has similar effect sizes to baseline cerebrospinal fluid (CSF) NfL [24].

We report results for convergent validity between BICAMS and ICA, test-retest reliability, correlation between ICA score and serum NfL, the effects of repeated exposure to the tests (i.e. learning bias), sensitivity to detecting cognitive impairment, and the accuracy of the ICA in discriminating MS patients from healthy controls (HC).

\section{Methods \\ ICA test description and the scientific rationale behind the test}

The ICA test is a rapid visual categorization task with backward masking [17, 18, 25]. The test takes advantage of the human brain's strong reaction to animal stimuli $[25,26]$. One hundred natural images (50 animal and 50 non-animal) are carefully selected, with varying levels of difficulty, and are presented to the participants in rapid succession. Images are presented in the center of the screen at a $7^{\circ}$ visual angle. In some images the head or body of the animal is clearly visible to the participants, which makes it easier to detect. In other images the animals are further away or otherwise presented in cluttered environments, making them more difficult to detect. A few sample images are shown in Fig. 1. We used grayscale images to remove the possibility of color blindness affecting participants' results. Furthermore, color images can facilitate animal detection solely based on color [27, 28], without full processing of stimulus shape. This could have made the task easier and less suitable for detecting less severe cognitive dysfunctions.

The strongest categorical division represented in the human higher level visual cortex appears to be that between animates and inanimates [29, 30]. Studies also show that on average it takes about $100 \mathrm{~ms}$ to $120 \mathrm{~ms}$ for the human brain to differentiate animate from inanimate stimuli [26, 31, 32]. Following this rationale, each image is presented for $100 \mathrm{~ms}$ followed by a 20 millisecond inter-stimulus interval (ISI), followed by a dynamic noisy mask (for $250 \mathrm{~ms}$ ), followed by the subject's categorization into animal vs. non-animal (Fig. 1). Shorter ISI durations can make the animal detection 


\section{ICA Test outline}

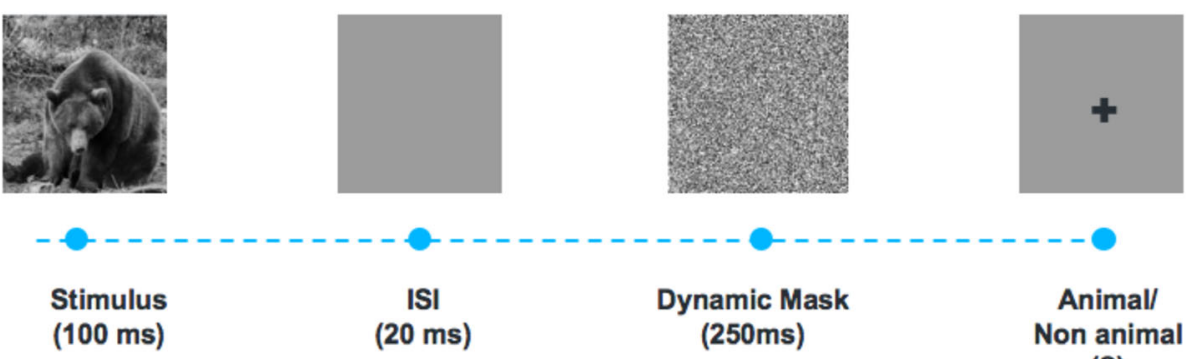

(?)

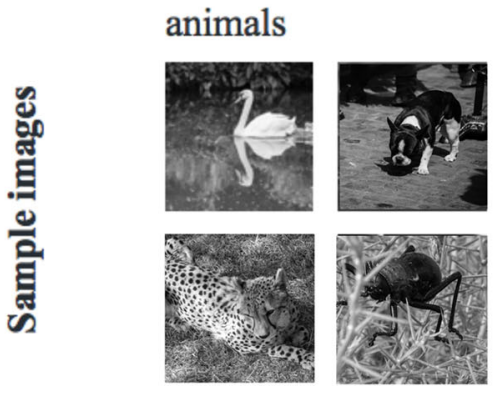

non-animals

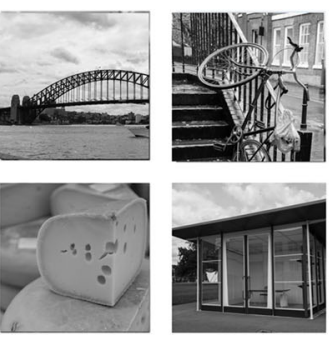

Fig. 1 The ICA test pipeline. One hundred natural images (50 animal and 50 non-animal) with various levels of difficulty are presented to the participants. Each image is presented for $100 \mathrm{~ms}$ followed by $20 \mathrm{~ms}$ inter-stimulus interval (ISI), followed by a dynamic noisy mask (for $250 \mathrm{~ms}$ ), followed by categorization into animal vs. non-animal. A few sample images are shown for demonstration purposes

task more difficult and longer durations reduce the potential use for testing purposes as it may not allow for the detection of less severe cognitive impairments. The dynamic mask is used to remove (or at least reduce) the effect of recurrent processes in the brain [33, 34]. This makes the task more challenging by reducing the ongoing recurrent neural activity that could artificially boost a subject's performance and further reduces the chances of learning the stimuli. For more information about rapid visual categorization tasks refer to Mirzaei et al., (2013) [17].

The ICA test starts with a different set of 10 test images ( 5 animal, 5 non-animal) to familiarize participants with the task. These images are later removed from further analysis. If participants perform above chance $(>50 \%)$ on these 10 images, they will continue to the main task. If they perform at chance level (or below), the test instructions will be presented again, and a new set of 10 introductory images will follow. If they perform above chance in this second attempt, they will progress to the main task. If they perform below chance for the second time the test is aborted.

\section{Backward masking}

To construct the dynamic mask a white noise image was filtered at four different spatial scales, and the resulting images were thresholded to generate high contrast binary patterns following the procedure in Bacon-Macé and colleagues (2005) [16, 17]. For each spatial scale, four new images were generated by rotating and mirroring the original image, creating a pool of 16 images. The noisy mask used in the ICA test was a sequence of $8 \mathrm{im}$ ages, chosen randomly from the pool, with each of the spatial scales appearing twice.

\section{Brief international cognitive assessment for MS (BICAMS)}

The BICAMS battery consists of three standard penand-paper tests, measuring speed of information processing, visuospatial learning and verbal learning.

\section{Symbol digit modalities test (SDMT)}

The SDMT is designed to assess speed of information processing, and takes about $5 \mathrm{~min}$ to administer [35]. The test is formed of a simple substitution task. Using a reference key, the examinee has $90 \mathrm{~s}$ to pair specific numbers with given geometric figures.

\section{California verbal learning test - 2nd edition (CVLT-II)}

The CVLT-II test $[36,37]$ measures episodic verbal learning. The test begins with the examiner reading a list of the 16 words. Participants listen to the list and then report as many of the items as they can recall. Five learning trials of the CVLT-II are used in BICAMS [20], which takes about $10 \mathrm{~min}$ to administer. 


\section{Brief visual memory test - revised (BVMT-R)}

The BVMT-R test assesses visuospatial learning (i.e. immediate recall) and memory (delayed recall) [38, 39]. Only learning trials of BVMT-R are included within BICAMS. Here, in three consecutive trials, six abstract shapes are presented to the participant for $10 \mathrm{~s}$. After each trial, the display is removed from view and patients are asked to draw the stimuli via pencil on paper manual responses. The test takes about 5 min to administer.

\section{Participants}

In total, 174 participants took part in Substudy 1 (Table 1): 91 patients diagnosed with multiple sclerosis (MS), and 83 healthy controls matched for age, gender and education. Fourty-eight MS patients took part in Substudy 2 (Table 2). Of all participants, 25 attended both substudies. Participants' age varied between 18 and 65. The study was conducted according to the Declaration of Helsinki and approved by the local ethics committee at Royan Institute. Informed written consent was obtained from all participants. Patient participants were consecutively recruited from the outpatient clinic of the Aria Medical Complex for MS in Tehran, Iran. Patients were diagnosed by a consultant neurologist according to the McDonald diagnostic criteria (2010 revision) [40]. Healthy controls (HC) were recruited through local advertisements.

Exclusion criteria: severe depression and other major psychiatric comorbidities, presence of neurological disorders and medical illness that independently affect brain function and cognition (other than MS for the patient group), visual problems that cannot be corrected with eye-glasses such that the problem prevents participants from reading, upper limb motor dysfunction, history of epileptic seizures, history of illicit substance and/ or alcohol dependence.

For each participant, the clinical characteristics of MS subtype, information on age, education and gender were

Table 1 Demographic and disease related information for participants in substudy 1

\begin{tabular}{llll}
\hline Characteristic & MS $(\boldsymbol{n}=\mathbf{9 1})$ & $\mathrm{HC}(\boldsymbol{n}=\mathbf{8 3})$ & $\boldsymbol{p}$-value \\
\hline Age -mean years \pm SD & $37.24 \pm 10.2$ & $36 \pm 10$ & 0.42 \\
Education in years -mean \pm SD & $14.21 \pm 3.16$ & $14.81 \pm 2.5$ & 0.16 \\
Gender (\%female) & $75(82 \%)$ & $58(70 \%)$ & 0.052 \\
Disease Duration (in years) & 6.8 & & \\
Disease course & & & \\
Relapsing remitting & $83(91 \%)$ & & \\
Secondary progressive & $6(7 \%)$ & & \\
Primary progressive & $2(2 \%)$ & & \\
EDSS -mean \pm SD & $1.27 \pm 1.8$ & & \\
\hline
\end{tabular}

$P$-values come from a two-sample t-test. MS: Multiple Sclerosis. HC: Healthy Controls. SD: standard deviation also collected. We quantified participant disability and disability progression over time by utilising the Expanded Disability Status Scale (EDSS).

For the purposes of this study, patients with severe abnormality in at least one of the BICAMS sub-tests (defined as 2 standard deviations (SD) below the norm) or with mild abnormality (defined as 1 SD below the norm) in at least two sub-tests of BICAMS were identified as cognitively impaired.

\section{Study procedures \\ Substudy 1}

One hundred seventy-four participants (Table 1) took the iPad-based ICA test and the pen-and-paper BICAMS test, administered in random order. The same researchers who administered the BICAMS directed participants on how to take the ICA test. In this substudy, we investigated convergent validity of the ICA test with BICAMS, ICA's test-retest reliability and the sensitivity and specificity of the ICA platform in detecting cognitive impairment in MS.

To measure test-retest reliability for the ICA test, a subset of $21 \mathrm{MS}$ and $22 \mathrm{HC}$ participants were called back after 5 weeks ( \pm 15 days) to take the ICA test as well as the SDMT. The subset's characteristics were similar to the primary set in terms of age, education and gender ratio. For both SDMT and the ICA, the same forms of the tests were used in the re-test session. Note that in the ICA test, while the images were the same, they were presented in a different random order in each administration.

\section{Substudy 2}

In this substudy, we investigated ICA's correlation with the level of serum NfL in 48 MS patients (Table 2). Participants took both the ICA and the SDMT test, administered in random order. The ICA and SDMT were administered in the same session, but blood samples were collected in another visit with a gap of $2-3$ days in between.

Blood samples were collected in tubes for serum isolation, then centrifuged at $3000 \mathrm{rpm}$ for $20 \mathrm{~min}$ of blood draw, and finally placed on ice. Serum samples were measured at 1:4 dilution. NfL concentrations in serum were measured using a commercial ELISA (NF-light ${ }^{\circ}$ ELISA, Uman Diagnostics, Umeå, Sweden). We used Anti NF-L monoclonal antibody $(\mathrm{mAB})$ as a capture antibody and a biotin-labeled Anti NF-L mAB as the detection antibody. All samples were measured blinded. ELISA readings were converted to units per milliliter by using a standard curve constructed by calibrators (Bovine lyophilized NfL obtained from UmanDiagnostics).

Participants in Substudy 2 also attended an 8-week physical and cognitive rehabilitation program, details of 
Table 2 Demographic and disease related information for participants in Substudy 2

\begin{tabular}{llccccc}
\hline Number of Participants & Disease Course & Age- mean years \pm SD & Gender (\%female) & EDSS- mean \pm SD & Education & Disease Duration \\
\hline 48 MS patients & Relapsing remitting & $34.12 \pm 8.5$ & $31(64 \%)$ & $2.95 \pm 0.92$ & $14.79 \pm 1.41$ & $8.27 \pm 5.20$ \\
\hline
\end{tabular}

which are reported in separate studies [41, 42]. The physical rehabilitation program included a combination of endurance and resistance exercises, with gradually increasing intensities over the 8-week period. The cognitive rehabilitation program included playing newlydeveloped games in a virtual reality (VR) environment, targeting sensorimotor integration, memory-based navigation and visual search. For the purpose of this study we measure pre- and post-rehabilitation ICA results for these group of participants, and the ICA correlation with NfL pre- and post-rehabilitation.

Participants were divided into a rehabilitation group of 38 individuals and a control group of ten; the control group only took the tests before and after these 8 weeks without attending the rehabilitation program. The rehabilitation group attended three sessions each week, each of them lasting about $70 \mathrm{~min}$.

\section{Accuracy, speed and ICA summary score calculations}

In the ICA, participants' responses to each image and their reaction times (i.e. time between image onset and response) are recorded and used to calculate their overall accuracy and speed. Speed and accuracy are then used to calculate an overall summary score, called the ICA score.

Accuracy is simply defined as the number of correct categorizations divided by the total number of images, multiplied by 100 .

$$
\begin{aligned}
\text { Accuracy }= & \frac{\text { number of correct categorizations }}{\text { total number of images }} \\
& \times 100
\end{aligned}
$$

Speed is defined based on participants' response reaction times to images they categorized correctly.

$$
\text { Speed }=\left[100,100 \times e^{\frac{- \text { mean correct } R T}{1025}+0.341}\right]
$$

\section{$R T$ : reaction time.}

e: Euler's number $2.7182 \ldots \ldots$

Speed is inversely related to reaction time; the higher the speed, the lower the reaction time.

\section{Preprocessing}

We used a boxplot to remove outlier reaction times, before computing the ICA score. A boxplot is a nonparametric method for describing groups of numerical data through their quartiles; and allows for detection of outliers in the data. Following the boxplot approach, reaction times greater than $\mathrm{q} 3+\mathrm{w} *(\mathrm{q} 3-\mathrm{q} 1)$ or less than $\mathrm{q} 1-\mathrm{w} *(\mathrm{q} 3-\mathrm{q} 1)$ are considered outliers (where $\mathrm{q} 1$ is the lower quartile, and q3 is the upper quartile of the reaction times; and " $w$ " is a 'whisker'; $w=1.5$ ). The number of reaction-time data points removed by the boxplot can vary case by case; if this number exceeds $40 \%$ of the observed images, the results are deemed invalid and a warning is shown to the clinician to repeat the test. In this study none of the participants faced such a warning. The maximum percentage of outliers was $15 \%$, which happened in one of the MS patients.

The ICA score is a combination of accuracy and speed, defined as follows:

$$
\text { ICA Score }=\left(\frac{\text { Speed }}{100} \times \frac{\text { Accuracy }}{100}\right) \times 100
$$

\section{ICA's artificial intelligence (AI) engine}

ICA's AI engine (Fig. 2) used in this study was a multinomial logistic regression (MLR) classifier trained based on a set of features extracted from the ICA test output for each participant. These features included the ICA score, and the trends of speed and accuracy during the test (i.e. whether the speed and/or accuracy were increasing or decreasing during the time-course of the test). The classifier also took subject's age, gender and education in order to match subjects with similar demographics.

Multinomial logistic regression classifier (MLR) [43] is a supervised regression-based learning algorithm. The learning algorithm's task is to learn a set of weights for a regression model that maps participants' ICA test output to classification labels.

The difference between ICA's AI engine in detecting cognitive impairment and the conventional way of defining a cut-off value for the outcome score of the test is further discussed in the discussion section.

\section{Results}

Convergent validity with BICAMS, and sensitivity to MS In Substudy 1, we assessed convergent validity by examining the correlation between scores on the ICA test and the BICAMS battery (i.e. SDMT, BVMT-R and CVLTII). Figure 3 presents scatterplots examining the relationship between BICAMS and ICA test performance. A high level of convergent validity is demonstrated between ICA and BICAMS. Within the BICAMS battery, SDMT had the highest correlation with the ICA test for 


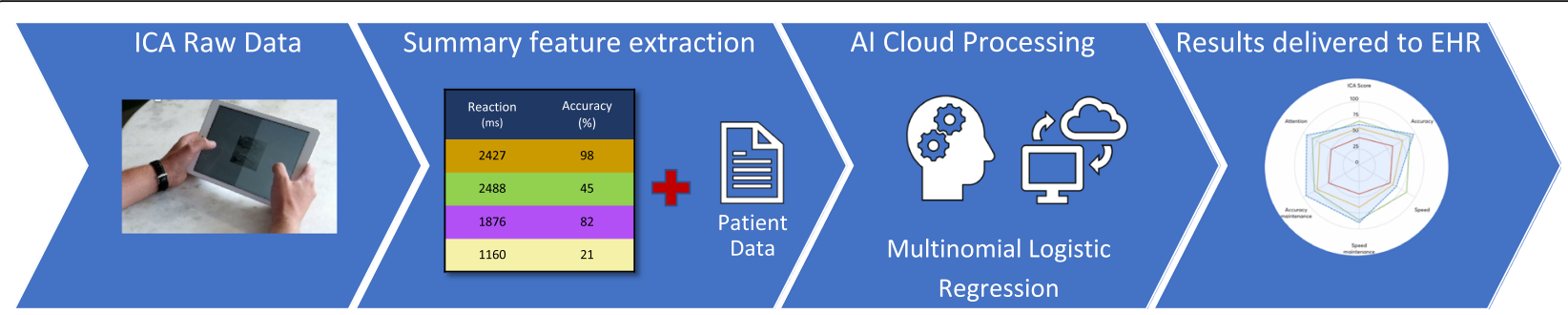

Fig. 2 ICA's Al's pathway. The ICA measures categorization accuracy, processing speed, accuracy and speed over time and the raw data from these measurements are combined with patients' demographic data, in order to provide a predictive score about participant's cognitive status. The above-mentioned extracted features from the ICA raw data, plus patient's demographic data are fed into an MLR classifier hosted on amazon AWS cloud services. The classifier then returns its predicted cognitive status, along with a probability, associated with the label, that shows how confident the Al engine is about the predicted label. The icons used in this figure are taken from Microsoft Office free icon library

the HC (Pearson's $\left.r=0.81, p<10^{-14}\right)$, MS $(r=0.71$, $\left.p<10^{-13}\right)$, and combined $\left(r=0.82, p<10^{-11}\right)$ groups. Scatterplots show ICA vs. BICAMS correlation separately for MS and $\mathrm{HC}$; combining results from both groups $(n=174$ total), we find a correlation of 0.82 with SDMT $\left(p<10^{-15}\right), 0.71$ with CVLT-II $(p<$ $\left.10^{-10}\right)$, and 0.60 with BVMT-R $\left(p<10^{-8}\right)$. The correlation results between BICAMS and ICA are largely similar when including only relapsing-remitting MS patients (RRMS) $[\mathrm{r} \quad(\mathrm{SDMT})=0.71 \quad(p<10-13) ; \mathrm{r}$ $($ BVMT-R $)=0.51 \quad(p<10-6) ; \mathrm{r} \quad($ CVLT-II $)=0.56 \quad(p<$ 10-7)]. Correlations between ICA's speed and accuracy components with the BICAMS battery are also reported in Table 3. Furthermore, we calculated BICAMS composite score by averaging the z-scores of the CVLT-II, the BVMT-R, and the SDMT. ICA had

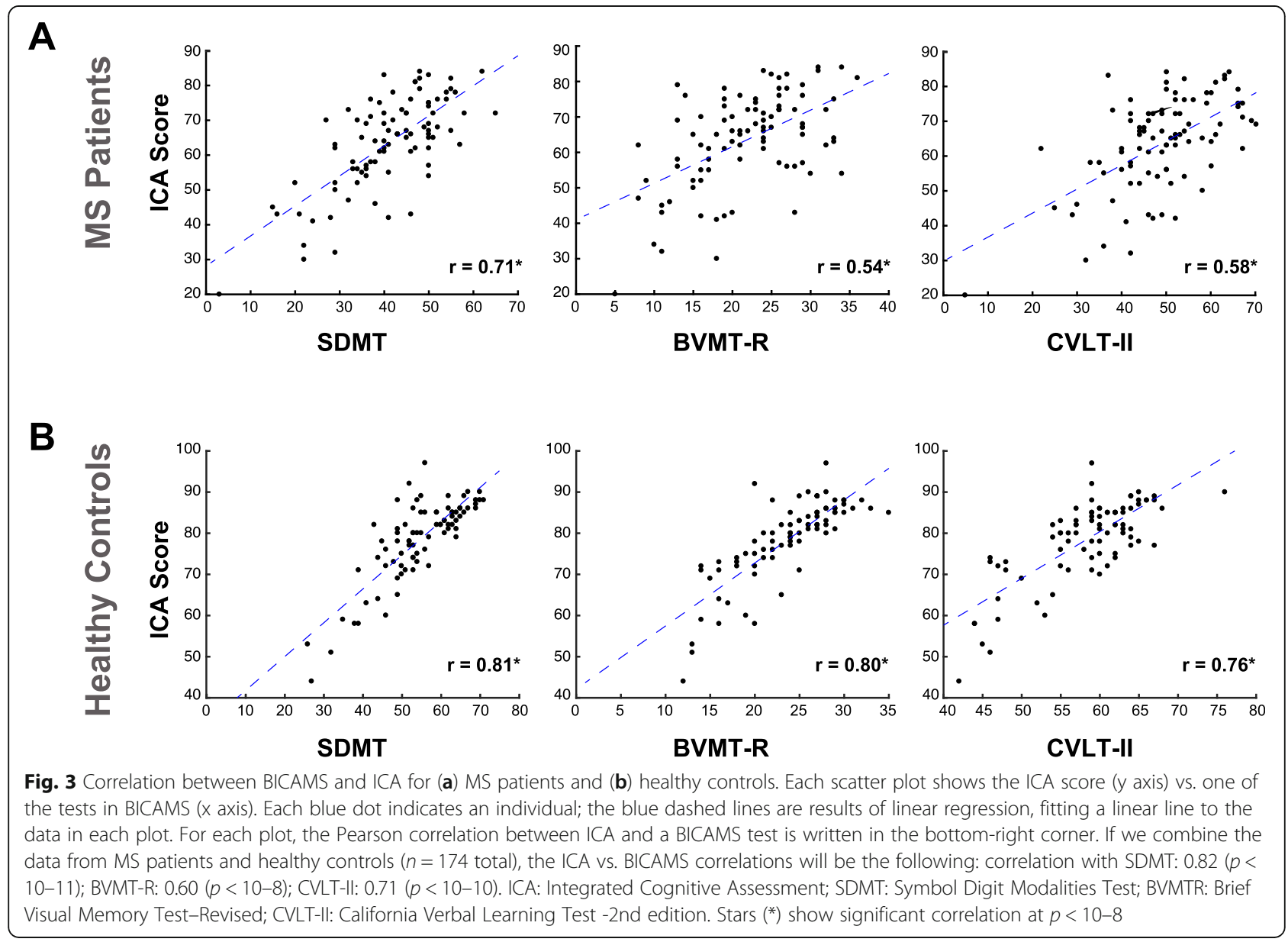


Table 3 Speed and accuracy correlations with BICAMS

\begin{tabular}{llll}
\hline & SDMT & BVMT-R & CVLT-II \\
\hline Speed & $r=0.66^{*}$ & $r=0.42^{*}$ & $r=0.52^{*}$ \\
Accuracy & $r=0.55^{*}$ & $r=0.46^{*}$ & $r=0.52 *$
\end{tabular}

Pearson correlations between the BICAMS battery and speed, accuracy components of the ICA test across all participants. (* shows statistical significance at $p<10^{-6}$ )

a correlation of $r=0.82\left(p<10^{-11}\right)$ with BICAMS composite score.

To compare the sensitivity of the BICAMS and ICA in detecting MS dysfunctions, we compared mean test scores in MS and HC groups separately for BICAMS battery of tests and the ICA test (Table 4). Within the BICAMS battery, SDMT and CVLT-II could differentiate between $\mathrm{HC}$ and MS patients (Table 4). The scores on both SDMT and CVLT-II were significantly lower for the MS patients compared to the HC group. However, there was no significant difference between BVMT-R scores of the HC and MS groups. These results are consistent with previous findings showing that SDMT has a better sensitivity in detecting MS compared to other tests within the BICAMS battery $[20,44]$. We repeated these analyses for the subset of RRMS patients (Supplementary Table 1), the results of which were similar to when all patients were included.

As shown in Table 4, the ICA could discriminate between $\mathrm{HC}$ group and MS patients, at least as accurately as the SDMT; however, the ICA, as a digital test, has the advantages described in Fig. 8.

Given that the ICA test involves tapping left or right on an iPad, we investigated the relation between handedness and ICA score. The correlation between ICA score and handedness was $r=-0.13, p=0.07$ (not significant), which is comparable (and lower) than the correlation between handedness and SDMT's score $(r=-0.17)$.

Table 4 Mean ICA and BICAMS scores per group

\begin{tabular}{|c|c|c|c|c|c|c|c|}
\hline \multirow[b]{2}{*}{ BICAMS } & \multicolumn{2}{|c|}{$\mathrm{MS}(\boldsymbol{n}=91)$} & \multicolumn{2}{|c|}{$\mathrm{HC}(n=83)$} & \multirow[b]{2}{*}{ Difference } & \multirow[b]{2}{*}{ Cohen's d } & \multirow[b]{2}{*}{$\boldsymbol{p}$-value } \\
\hline & mean & SD & mean & SD & & & \\
\hline SDMT & 41.04 & 11.02 & 54.73 & 9.77 & 13.69 & 1.31 & $<10^{-14}$ \\
\hline BVMT-R & 21.89 & 6.95 & 23.69 & 5.17 & 1.80 & 0.29 & $=0.0565$ \\
\hline CVLT-II & 48.96 & 11.13 & 58.28 & 6.59 & 9.32 & 1.00 & $<10^{-9}$ \\
\hline \multicolumn{8}{|l|}{ ICA } \\
\hline ICA score & 63.67 & 13.30 & 78.43 & 9.86 & 17.76 & 1.26 & $<10^{-13}$ \\
\hline Accuracy & 84.97 & 11.66 & 89.57 & 5.79 & 4.60 & 0.50 & $=0.0014$ \\
\hline Speed & 74.54 & 12.26 & 87.76 & 10.11 & 13.22 & 1.16 & $<10^{-12}$ \\
\hline
\end{tabular}

Mean and standard deviations (SD) for BICAMS test scores and the ICA test scores are compared for MS patients versus healthy controls (HC). The ICA score is a composite score made of both speed and accuracy of participants in ICA's rapid visual categorization task. $P$-values come from a two-sample t-test

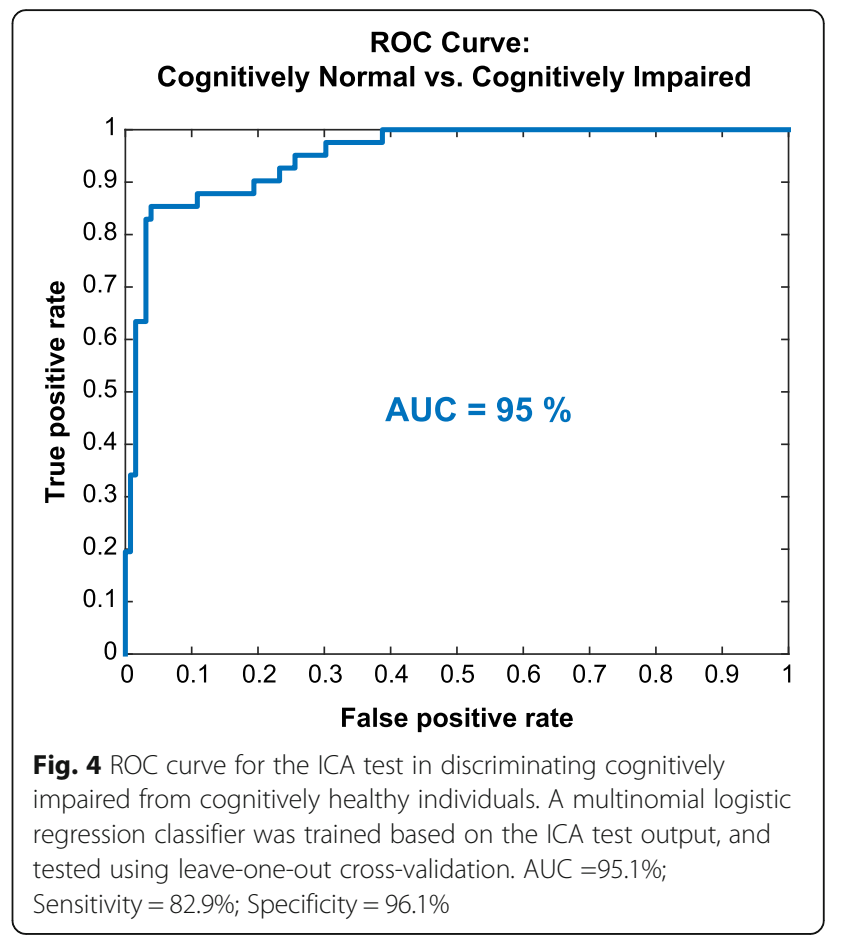

\section{ICA accuracy in detecting cognitive impairment}

$45 \%$ of MS patients were identified to have cognitive impairment. Using an ROC curve (Fig. 4), we then assessed the accuracy of the ICA's AI engine (i.e. MLR classifier) in discriminating cognitively healthy from cognitively impaired individuals (Fig. 4, area under curve (AUC) = $95.1 \%$, sensitivity $=82.9 \%$, and specificity $=96.1 \%$.)

\section{ICA and SDMT correlations with Neurofilament light (NfL)} $\mathrm{NfL}$ is a promising fluid biomarker of disease monitoring for various brain disorders, such as Alzheimer's Disease and Multiple Sclerosis $[45,46]$. In Substudy 2, we demonstrated that there is a strong correlation between ICA score and the level of serum NfL $\left(r=-0.79, p<10^{-10}\right)$ (Fig. 5a). For comparison, on the same set of MS participants, SDMT correlations with NfL is also reported $\left(r=-0.67, p<10^{-6}\right)$ (Fig. 5b). SDMT and ICA were both administered in the same session.

\section{ICA's performance pre- and post- rehabilitation}

We examined the level of serum NfL pre- and post- rehabilitation, as well as patients' EDSS and ICA scores (Fig. 6). In the rehabilitation group, after the 8-week rehabilitation program, we observed a significant increase in ICA score (Cohen's $\mathrm{d}=0.8, p<0.0001$ ), and a significant decrease in serum-NfL $(\mathrm{d}=-0.4, p<0.01)$ and EDSS score $(\mathrm{d}=-0.4, p<0.01)$. In the control group, we found the opposite pattern after 8 weeks, that is a decrease in ICA score $(\mathrm{d}=-0.4, p>0.05)$ and a significant 
A

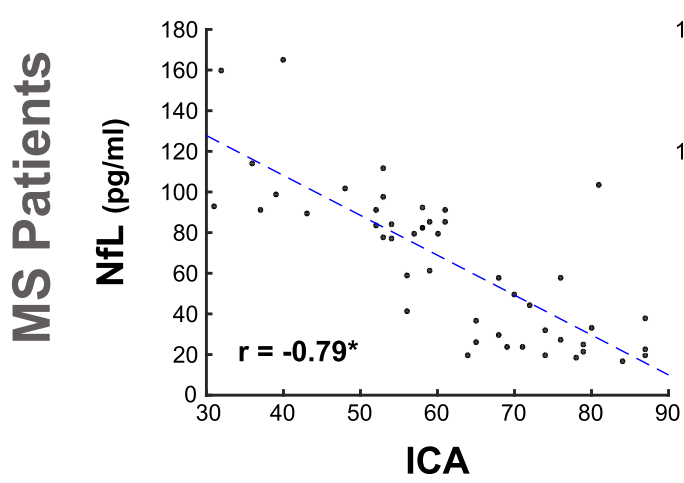

B

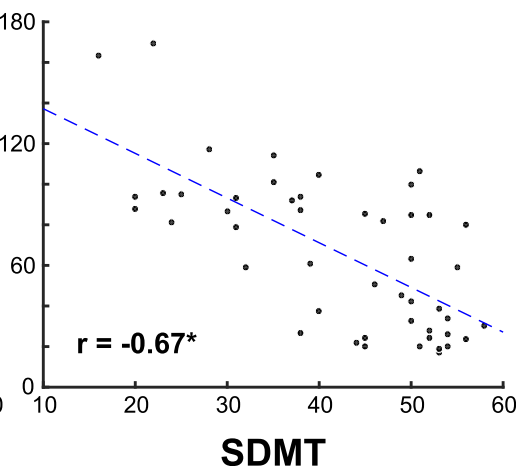

Fig. 5 ICA correlation with severity of neural damage, as measured by serum NfL. Each scatter plot shows the NfL level in serum (y axis) vs. ICA or SDMT (x axis). Each blue dot indicates an individual; the blue dashed lines are results of linear regression, fitting a linear line to the data in each plot. For each plot, the Pearson correlation between NfL level and the reference cognitive test is written in the bottom-left. Stars (*) show significant correlations at $p<10^{-6}$

increase in serum-NfL $(\mathrm{d}=0.9, p<0.001)$ and EDSS score $(\mathrm{d}=1.0, p<0.03)$.

\section{ICA test-retest reliability and absence of a learning bias}

Test-retest reliability was measured by computing the Pearson correlation between the two ICA scores. R values for test-retest correlation are considered adequate if $>0.70$ and good if $>0.80$ [47].
Figure 7 presents scatterplots of ICA performance comparing 1st administration versus 2 nd administration of the test for the HC, MS, and combined groups. Testretest reliability was high, with correlation values in the range between 0.91 and 0.94 .

In the subgroup of participants (21 MS and $22 \mathrm{HC}$ ) who took the ICA and SDMT for a second time, we studied whether they could perform better because of a previous exposure to either of the tests. This is called a

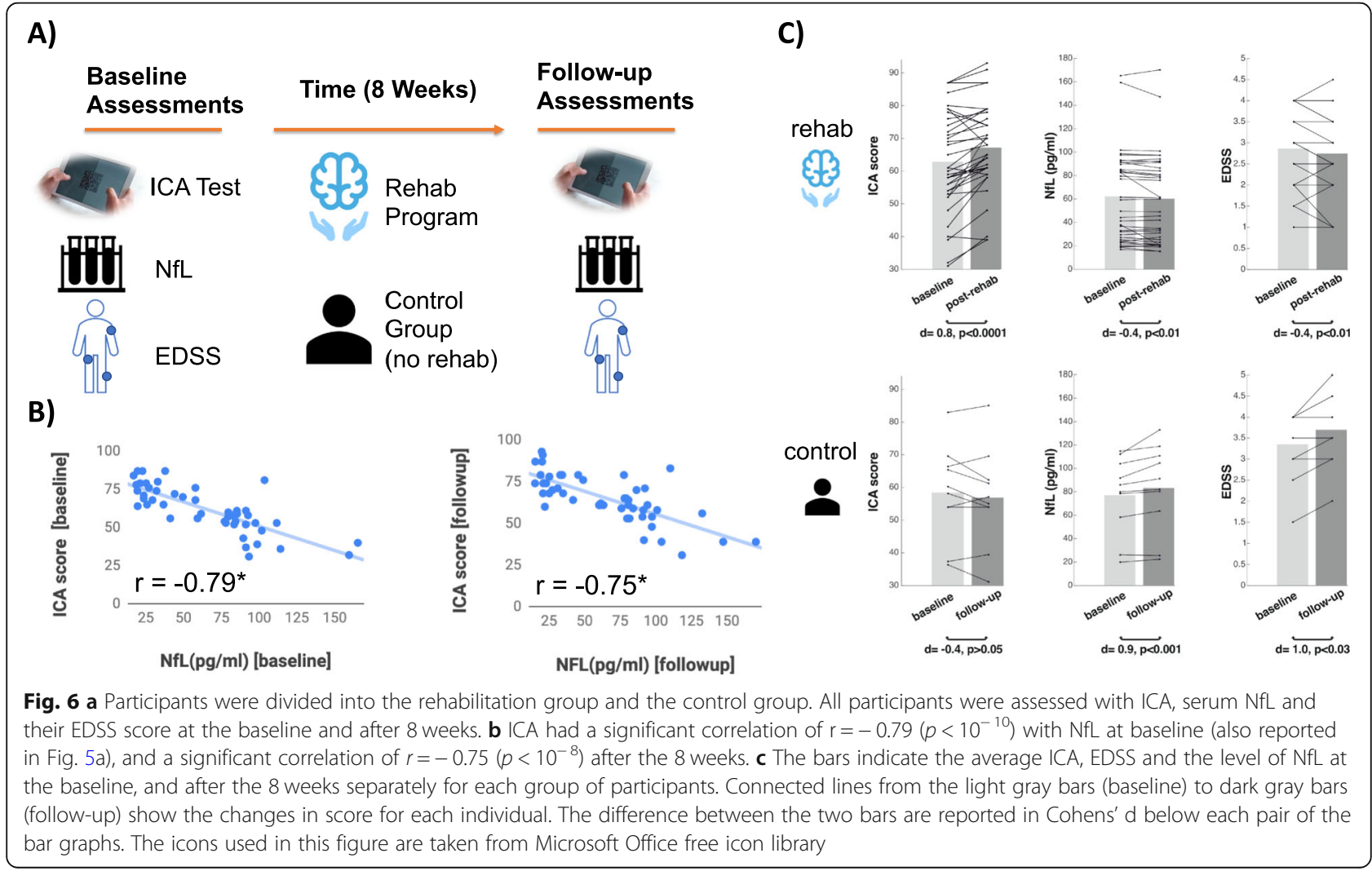



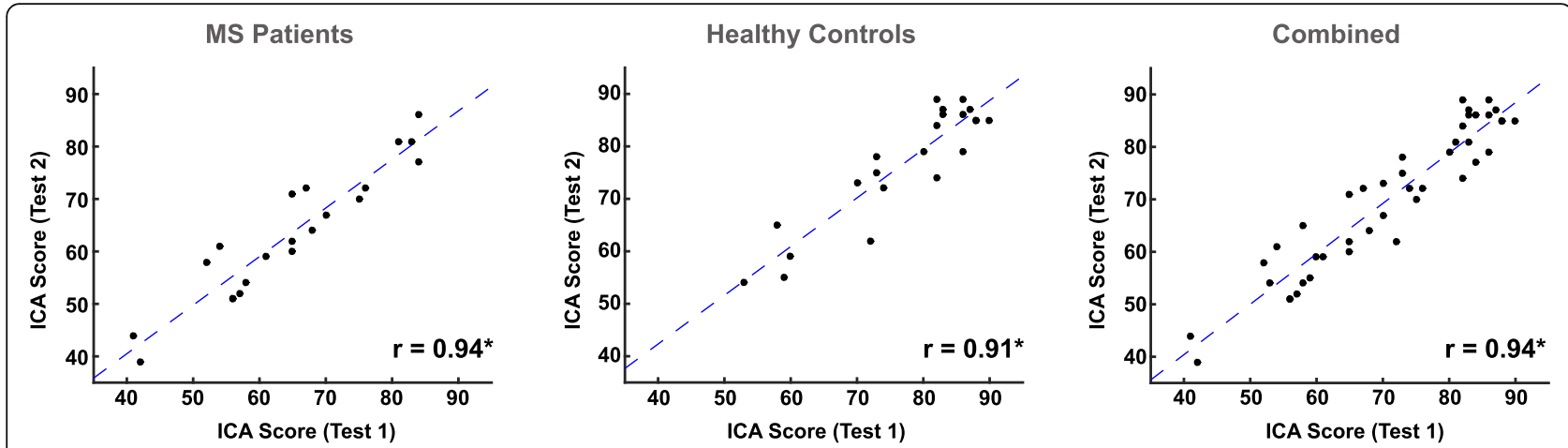

Fig. 7 Test-retest reliability scatter plots for the ICA test. Scatterplots are presented comparing ICA scores at Time 1 versus Time 2 administrations for the MS, HC, and combined groups. The gap between the 1st and the 2nd administration of the ICA test was 5 weeks (+ -15 days). Reliability is calculated using Pearson's $r$. The test-retest reliability for the SDMT test was: $r$ (combined) $=0.97 ; r(H C)=0.98 ; r(M S)=0.97$. Stars $\left(^{*}\right)$ indicate statistical significance at $p<10^{-8}$

learning bias (also referred to as a practice effect). As shown in Table 5, comparing the first and second administration of the ICA and SDMT tests, ICA showed no learning bias. However, we see an improvement in participants' average SDMT score. This improvement in SDMT score (i.e. learning bias) was statistically significant in the HC group, but not in the MS group.

\section{ICA correlation with EDSS, age and education}

To further characterize the ICA score and its relationship with other measures from the MS patients, we calculated the correlation between ICA score and patients' EDSS, age and education (Table 6). Both BICAMS and ICA scores were negatively correlated with patients' EDSS, demonstrating an inverse relation between disability scale and cognitive performance. For all the tests, we also observed a decrease in performance as age increased, showing the effect of aging on cognitive performance. All tests were correlated with participants' level of education, with ICA having the lowest correlation.

\section{Discussion}

In this validation study, we demonstrate that the ICA test has convergent validity with BICAMS, with an excellent test-retest reliability comparable to that reported for SDMT [10]. In the ICA test, comparing speed versus accuracy (Table 4), speed seems to play a more significant role in discriminating MS patients from $\mathrm{HC}$ participants. This corroborates findings from other studies suggesting slower speed of information processing as a key deficit in multiple sclerosis [48]. IPS impairment also underlies other areas of cognitive dysfunction $[15,49]$. This is because the speed with which an individual performs a cognitive task is not simply an isolated function of the processes required in that task, but also a reflection of their ability to rapidly carry out many different types of processing operations. In the case of ICA, these operations include transferring visual information through retina to higher level visual areas (i.e. sensory speed), processing the image representation in the visual system to categorize it into animal or non-animal (i.e. cognitive speed), and then translating this into a motor response (i.e. motor speed).

We also explored the link between disability (as measured by EDSS) and cognitive impairment. Patients with cognitive impairment are typically found to be at higher risk of developing further disability $[6,8]$. While we did not carry out a long-term monitoring of disability progression in our patients in this study, the negative correlation between ICA score and EDSS score

Table 5 Learning bias (practice effect) for ICA and SDMT

\begin{tabular}{|c|c|c|c|c|c|c|}
\hline \multirow[t]{2}{*}{ Group } & \multirow{2}{*}{$\begin{array}{l}\text { Test } \\
\text { Name }\end{array}$} & \multirow{2}{*}{$\begin{array}{l}\text { Test } 1 \\
{[\text { mean } \pm \text { SD] }}\end{array}$} & \multirow{2}{*}{$\begin{array}{l}\text { Test } 2 \\
{[\text { mean } \pm \text { SD] }}\end{array}$} & \multicolumn{3}{|c|}{ Paired t-test } \\
\hline & & & & difference & Cohen's d & $\boldsymbol{p}$-value \\
\hline \multirow[t]{2}{*}{ MS $(n=21)$} & ICA & $64.76 \pm 12.7$ & $64.66 \pm 12.2$ & -0.09 & -0.02 & 0.91 \\
\hline & SDMT & $43.6 \pm 10$ & $44.2 \pm 11$ & 0.66 & 0.25 & 0.25 \\
\hline \multirow[t]{2}{*}{$\mathrm{HC}(n=22)$} & ICA & $77.04 \pm 11$ & $76.86 \pm 11$ & -0.18 & -0.07 & 0.73 \\
\hline & SDMT & $55.13 \pm 12$ & $56.36 \pm 12.3$ & 1.22 & 0.50 & $0.02^{*}$ \\
\hline
\end{tabular}

Mean and standard deviations (SD) of SDMT and ICA scores are compared between the two administrations of the tests for each group of participants. Only SDMT in healthy subjects showed a significant learning bias 
Table 6 Age/EDSS/Education vs. BICAMS/ICA

\begin{tabular}{|c|c|c|c|c|c|}
\hline & & \multicolumn{3}{|l|}{ BICAMS } & \multirow[b]{2}{*}{ ICA } \\
\hline & & $\overline{\mathrm{SDMT}}$ & BVMT-R & CVLT-II & \\
\hline \multirow[t]{3}{*}{ Correlation with } & EDSS & $-0.41\left(p<10^{-4}\right)$ & $-0.26(p<0.05)$ & $\begin{array}{l}-0.33 \\
(p<0.001)\end{array}$ & $-0.58\left(p<10^{-8}\right)$ \\
\hline & Education & $0.50\left(p<10^{-6}\right)$ & $\begin{array}{l}0.34 \\
(p<0.001)\end{array}$ & $\begin{array}{l}0.31 \\
(p<0.01)\end{array}$ & $0.25(p<0.05)$ \\
\hline & Age & $-0.38\left(p<10^{-4}\right)$ & $\begin{array}{l}-0.50 \\
\left(p<10^{-5}\right)\end{array}$ & $\begin{array}{l}-0.42 \\
\left(p<10^{-4}\right)\end{array}$ & $-0.49\left(p<10^{-6}\right)$ \\
\hline
\end{tabular}

The table shows Pearson Correlations of the ICA score and the BICAMS battery of tests with MS patients' EDSS score, education in years, and their age. EDSS Expanded Disability Status Scale; BICAMS Brief International Cognitive Assessment for MS; SDMT Symbol Digit Modalities Test; BVMT-R Brief Visual Memory TestRevised; CVLT-I/ California Verbal Learning Test -2nd edition; ICA Integrated Cognitive Assessment

corroborates previous findings that lower cognitive performance is linked with higher disability (i.e. as indicated by higher EDSS scores).

In contrast to most of the currently standard cognitive tests, whereby stimuli are language-dependent, the presented stimuli in the ICA test are natural images that contain universally recognizable images of animals or objects, thus making the test intrinsically languageindependent. Furthermore, participants' responses only involve tapping on the left or right side of an iPad, making it totally independent of participants' knowledge of Arabic numerals or alphabet and words, or ability of a participant to draw shapes (as in BVMT-R). This makes the ICA test more suitable for wider international use, and less dependent on linguistic, educational, and demographic differences.

Computerized tests have several advantages over penand-paper tests, such as a) efficient administration that can save expensive clinical time, b) automatic scoring, which reduces errors in calculating and transferring scores, and c) easier integration with electronic medical records or research databases. The use of digital technology in this context can reduce barriers for both clinicians and patients to deliver or receive the assessments that would benefit their treatment and health throughout the course of the disease. With ICA, we aimed to develop a test that can close the current gap in clinical practice between patients' needs and what clinicians can offer in terms of the much-needed routine cognitive assessment and disease monitoring. Such a test must have a certain set of attributes, in addition to being sensitive and accurate. Figure 8 summarizes some of the key attributes of the ICA test, as a computerized test, that has made it more scalable, accessible and costeffective compared to the standard pen-and-paper tests. In addition, the ICA has some unique features that are absent from the computerized versions of the standard cognitive tests, such as the ability to benefit from more data to improve its performance over time. One basic difference between the ICA's classification of patients (using the AI engine) and the conventional way of defining an optimal cut-off value for classification is the dimensionality (or the number of features) used to make the classification. For example, in a conventional assessment tool, an optimal cut-off value is defined based on the test score. This is a one-dimensional classification

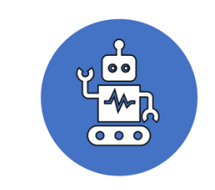

EMPLOYMENT OF AI (CAN BENEFIT FROM MORE DATA)

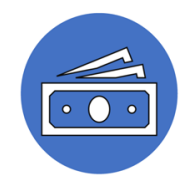

COST EFFECTIVE

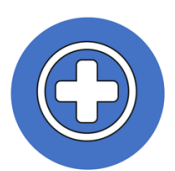

EASIER INTEGRATION WITH ELECTRONIC HEALTH RECORDS

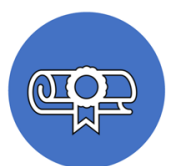

NOT BIASED BY EDUCATION

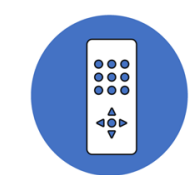

CAN ENABLE REMOTE MONITORING

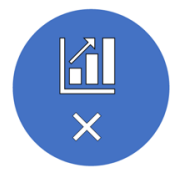

NO PRACTICE EFFECT

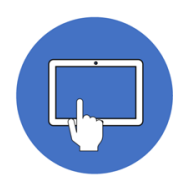

SELF

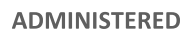

\&

USER-FRIENDLY

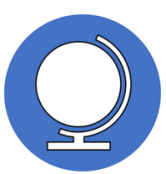

LANGUAGE INDEPENDENCE

Fig. 8 ICA key features as a computerized test. Eight key attributes of the ICA test that can save expensive clinical time and make the test scalable and more accessible to wider populations; as well as its capability to use new data as they become available to improve its reliability over time. The icons used in this figure are taken from Microsoft Office free icon library 
problem, and there is only one free parameter to optimize, resulting in less flexibility to learn from more data. In contrast, the ICA generates a richer pool of data (one reaction time and accuracy result per image). Although the ICA score is the most informative summary score, the use of a classifier enabled us to find the optimum classification boundary in the higher dimensional space. With more free parameters to optimize, the classifier benefited from more data to set these parameters to achieve a higher classification accuracy. The ICA's performance can be further improved over time through new batches of training on additional data to update its cloud-based AI model.

It is worth noting that while electronic implementations of the SDMT $[50,51]$ do exist, they retain two significant differences from the ICA. First, the ICA takes advantage of an AI platform, and thus the capacity to benefit from big data, as explained before. Second, the ICA did not show a learning bias in this and a previous study [19], as opposed to the learning bias reported for the iPad-based SDMT (i.e. PST) [50].

To make an early diagnosis of MS and monitor disease activity, reliable biomarkers are required. In Substudy 2, we demonstrated a strong association between ICA score and NfL in MS patients. This is particularly of interest given the totally non-invasive nature of the ICA test, and its easy and inexpensive scalability for administration in large populations. The 8-week follow-up of the rehabilitation group, compared to the control group, further shows ICA's sensitivity to track changes in cognition. For frequent cognitive assessments, digital biomarkers have an advantage over fluid biomarkers, given their lower cost, accessibility, the possibility of remote administration and easier integration into routine clinical practice.

Limitations encountered in this study include the lack of NfL data from the healthy control group, and the absence of neuroimaging markers of disease activity. We acknowledge the relatively short follow-up period of patients post-rehabilitation. Future studies are needed to investigate the link between ICA test results and other measures of brain atrophy, in particular, given the strong link between ICA and NfL-which reflects neural damage-would be informative to investigate the ICA relation with cortical thickness in MS patients.

\section{Conclusions}

Overall, our results provide evidence for the use of the ICA in clinical practice as an accurate tool for assessing cognitive impairment in MS. Digital biomarkers of cognition (such as ICA) can be used to monitor the progress of cognitive impairment, which subsequently paves the way for using cognition as a marker of disease activity in MS. The ICA has the potential to be used as a high-frequency monitoring tool of treatment efficacy both in the clinic and remotely such as at patients' homes. Future longitudinal studies need to test these hypotheses in larger patient samples.

\section{Supplementary information}

Supplementary information accompanies this paper at https://doi.org/10. 1186/s12883-020-01736-x

Additional file 1. Supplementary Table 1 Mean ICA and BICAMS scores for RRMS and HC

\section{Abbreviations}

MS: Multiple sclerosis; Al: Artificial intelligence; ICA: Integrated cognitive assessment; BICAMS: Brief international cognitive assessment for multiple sclerosis; HC: Healthy controls; NfL: Neurofilament light; IPS: Information processing speed; SDMT: Symbol digit modalities test; PST: Processing speed test; CVLT-II: California verbal learning test -2nd edition; BVMT-R: Brief visual memory test-revised; ISI: Inter-stimulus interval

\section{Acknowledgements}

We thank James Fraser and Hadi Modarres for reviewing and editing the paper prior to submission. We thank Vladyslav Ogol and other members of the ONSEO development team, for their great help and contribution in developing the ICA software needed for data acquisitions. We would also like to thank Jonathan El-Sharkawy for his help in creating and designing some of the Figures.

\section{Authors contributions}

SMN and SMKR and CK conceived and designed the study. SMN, MS, and MK did the data collection and patient recruitment. The data were analyzed by MS and MK, under the supervision of MSN and SMKR. All authors were involved in writing the manuscript. All authors have read and approved the manuscript.

\section{Funding}

SMKR was funded by a return home fellowship grant from the Iranian National Elite Foundation. Cognetivity Itd covered the costs for purchasing BICAMS. Other costs were covered by the Royan Institute internal funds to the investigators SMN, and SMKR. As stated in the competing interests section, the authors SMKR and CK are affiliated with one of the funding sources; other than that the funding sources had no direct role or any contractual forces to intervene in the design of the study, data collection, analyses, interpretation of the data, writing the manuscript or decision to submit the results.

\section{Availability of data and materials}

De-identified raw data are available to qualified investigators upon reasonable request from the corresponding author for the purposes of replicating procedures and results.

\section{Ethics approval and consent to participate}

The study was conducted according to the Declaration of Helsinki and approved by the Royan Institute's ethics committee (ref: IR.ACECR.ROYAN. REC.IR.ACECR.ROYAN.REC.1396.98). Informed written consent was obtained from all participants.

\section{Consent for publication}

Not applicable.

\section{Competing interests}

SMKR serves as the chief science officer at Cognetivity Itd; CK serves as Chief Medical Officer at Cognetivity Ltd. Other authors declared no potential conflicts of interest.

\section{Author details}

${ }^{1}$ Cognetivity Ltd, London, UK. ²Department of Brain and Cognitive Sciences, Cell Science Research Center, Royan Institute for Stem Cell Biology and 
Technology, ACECR, Tehran, Iran. ${ }^{3}$ University of Tehran, Tehran, Iran. ${ }^{4}$ South London \& Maudsley NHS Foundation Trust, London, UK. ${ }^{5}$ Department of Old Age Psychiatry, King's College London, London, UK.

Received: 29 May 2019 Accepted: 20 April 2020

Published online: 18 May 2020

\section{References}

1. Trapp BD, Nave K-A. Multiple sclerosis: an immune or neurodegenerative disorder? Annu Rev Neurosci. 2008;31:247-69.

2. Filippo MD, Portaccio E, Mancini A, Calabresi P. Multiple sclerosis and cognition: synaptic failure and network dysfunction. Nat Rev Neurosci. 2018; 19:599-609.

3. Rao SM, et al. Cognitive dysfunction in multiple sclerosis. II Impact on employment and social functioning. Neurology. 1991;41:692-6.

4. Schultheis MT, et al. Examining the relationship between cognition and driving performance in multiple sclerosis. Arch Phys Med Rehabil. 2010;91: 465-73

5. Foley FW, Benedict RH, Gromisch ES, DeLuca J. The need for screening, assessment, and treatment for cognitive dysfunction in multiple sclerosis: results of a multidisciplinary CMSC consensus conference, September 24, 2010. Int J MS Care. 2012;14:58-64

6. Pitteri M, Romualdi C, Magliozzi R, Monaco S, Calabrese M. Cognitive impairment predicts disability progression and cortical thinning in MS: an 8year study. Mult Scler J. 2017;23:848-54.

7. Sumowski JF, et al. Cognition in multiple sclerosis: State of the field and priorities for the future. Neurology. 2018;90(6):278-88. https://www.ncbi.nIm. nih.gov/pubmed/29343470.

8. Motyl, J. et al. Cognition as a Disability Progression Marker: Two-Years FollowUp of People with Multiple Sclerosis (P5. 2-015). (AAN Enterprises, 2019).

9. Benedict $\mathrm{RH}$. Effects of using same-versus alternate-form memory tests during short-interval repeated assessments in multiple sclerosis. J Int Neuropsychol Soc. 2005;11:727-36.

10. Benedict RH, et al. Repeated assessment of neuropsychological deficits in multiple sclerosis using the symbol digit modalities test and the MS neuropsychological screening questionnaire. Mult Scler J. 2008;14:940-6.

11. Calabresi PA, et al. Serum neurofilament light (NfL): towards a blood test for prognosis and disease/treatment monitoring in multiple sclerosis patients (S24.003). Neurology. 2018;90:S24.003.

12. Eshaghi $A$, et al. Progression of regional grey matter atrophy in multiple sclerosis. Brain. 2018;141:1665-77.

13. Comabella M, Montalban X. Body fluid biomarkers in multiple sclerosis. Lancet Neurol. 2014;13:113-26.

14. Qureshi SS, Beh SC, Frohman TC, Frohman EM. An update on neuroophthalmology of multiple sclerosis: the visual system as a model to study multiple sclerosis. Curr Opin Neurol. 2014;27:300-8.

15. Costa SL, Genova HM, DeLuca J, Chiaravalloti ND. Information processing speed in multiple sclerosis: past, present, and future. Mult Scler Houndmills Basingstoke Engl. 2017;23:772-89.

16. Bacon-Macé N, Macé MJ-M, Fabre-Thorpe M, Thorpe SJ. The time course of visual processing: backward masking and natural scene categorisation. Vis Res. 2005;45:1459-69.

17. Mirzaei A, Khaligh-Razavi S-M, Ghodrati M, Zabbah S, Ebrahimpour R. Predicting the human reaction time based on natural image statistics in a rapid categorization task. Vis Res. 2013:81:36-44.

18. Khaligh-Razavi, S.-M. \& Habibi, S. System for assessing a mental health disorder. (2016)

19. Khaligh-Razavi S-M, Habibi S, Sadeghi E, Kalafatis C. Integrated cognitive assessment: speed and accuracy of visual processing as a proxy to cognitive performance. J Vis. 2018;18:104

20. Benedict $\mathrm{RH}$, et al. Brief international cognitive assessment for MS (BICAMS): international standards for validation. BMC Neurol. 2012;12:55.

21. Langdon DW, et al. Recommendations for a brief international cognitive assessment for multiple sclerosis (BICAMS). Mult Scler J. 2012;18:891-8.

22. Berger T, Stüve $\mathrm{O}$. Neurofilament light chain: an important step toward a disease biomarker in multiple sclerosis. Neurology. 2019;92:451-2.

23. Kuhle J, et al. Blood neurofilament light chain as a biomarker of MS disease activity and treatment response. Neurology. 2019;92:e1007-15.

24. Mielke MM, et al. Plasma and CSF neurofilament light: Relation to longitudinal neuroimaging and cognitive measures. Neurology. 2019;93(3):e252-e260. https://www.ncbi.nlm.nih.gov/pubmed/31182505.
25. Kiani R, Esteky H, Mirpour K, Tanaka K. Object category structure in response patterns of neuronal population in monkey inferior temporal cortex. J Neurophysiol. 2007;97:4296-309.

26. Cichy RM, Pantazis $D$, Oliva A. Resolving human object recognition in space and time. Nat Neurosci. 2014;17:455-62.

27. Marx S, Hansen-Goos O, Thrun M, Einhäuser W. Rapid serial processing of natural scenes: color modulates detection but neither recognition nor the attentional blink. J Vis. 2014;14:4.

28. Zhu W, Drewes J, Gegenfurtner KR. Animal Detection in Natural Images: Effects of Color and Image Database. PLoS ONE. 2013;8(10):e75816. https://doi.org/10.1371/journal.pone.0075816.

29. Kriegeskorte $\mathrm{N}$, et al. Matching categorical object representations in inferior temporal cortex of man and monkey. Neuron. 2008;60:1126-41.

30. Naselaris T, Stansbury DE, Gallant JL. Cortical representation of animate and inanimate objects in complex natural scenes. J Physiol-Paris. 2012: 106:239-49

31. Liu H, Agam Y, Madsen JR, Kreiman G. Timing, timing, timing: fast decoding of object information from intracranial field potentials in human visual cortex. Neuron. 2009:62:281-90.

32. Khaligh-Razavi S-M, Cichy RM, Pantazis D, Oliva A. Tracking the spatiotemporal neural dynamics of real-world object size and Animacy in the human brain. J Cogn Neurosci. 2018;30:1559-76.

33. Fahrenfort JJ, Scholte HS, Lamme VAF. Masking disrupts reentrant processing in human visual cortex. J. Cogn. Neurosci. 2007;19: 1488-97.

34. Rajaei K, Mohsenzadeh Y, Ebrahimpour R, Khaligh-Razavi S-M. Beyond core object recognition: recurrent processes account for object recognition under occlusion. PLoS Comput Biol. 2019;15:e1007001

35. Smith A. Symbol digit modalities test. CA: Western Psychological Services Los Angeles; 1982

36. Yi A. California verbal learning test (California Verbal Learning TestII). In: Kreutzer JS, DeLuca J, Caplan B, editors. Encyclopedia of Clinical Neuropsychology. New York: Springer; 2011. https://link. springer.com/referenceworkentry/10.1007\%2F978-0-387-79948-3 1112 \#howtocite.

37. Stegen $\mathrm{S}$, et al. Validity of the California verbal learning test-II in multiple sclerosis. Clin Neuropsychol. 2010;24:189-202.

38. Benedict RHB, Schretlen D, Groninger L, Dobraski M, Shpritz B. Revision of the brief Visuospatial memory test: studies of normal performance, reliability, and validity. Psychol Assess. 1996:8:145-53.

39. Benedict RHB, Schretlen D, Groninger L, Dobraski M, Shpritz B. Revision of the Brief Visuospatial Memory Test: Studies of normal performance, reliability, and validity. Psychological Assessment. 1996;8(2);145-53. https://doi.org/10.1037/1040-3590.8.2.145

40. Polman $\mathrm{CH}$, et al. Diagnostic criteria for multiple sclerosis: 2010 revisions to the McDonald criteria. Ann Neurol. 2011;69:292-302.

41. Khaligh-Razavi S-M, Sadeghi M, Khanbagi M, Kalafatis C, Nabavi SM. Using ICA-an artificial intelligence (Al)-assisted technology-as a digital biomarker of MS disease progression and treatment efficacy. Mult Scler J. 2019;25:722-3.

42. Sadeghi $M$, et al. Virtual reality (VR)-based cognitive rehabilitation: cognitive games are complementary to physical training for an optimum rehabilitation strategy in patients with multiple sclerosis (MS). Mult Scler J. 2019;25:66-6.

43. Hastie T, Tibshirani R, Friedman J. The elements of statistical learning. New York: Springer; 2009

44. Eshaghi A, et al. Validity and reliability of a Persian translation of the minimal assessment of cognitive function in multiple sclerosis (MACFIMS). Clin Neuropsychol. 2012;26:975-84

45. Lewczuk $P$, et al. Plasma neurofilament light as a potential biomarker of neurodegeneration in Alzheimer's disease. Alzheimers Res Ther. 2018;10:71

46. Cai L, Huang J. Neurofilament light chain as a biological marker for multiple sclerosis: a meta-analysis study. Neuropsychiatr Dis Treat. 2018; 14:2241-54.

47. Anastasi A. Psychological testing. 6th ed. New York: Macmillan Publishing Company; 1988

48. Demaree HA, DeLuca J, Gaudino EA, Diamond BJ. Speed of information processing as a key deficit in multiple sclerosis: implications for rehabilitation. J Neurol Neurosurg Psychiatry. 1999:67:661-3. 
49. DeLuca J, Chelune GJ, Tulsky DS, Lengenfelder J, Chiaravalloti ND. Is speed of processing or working memory the primary information processing deficit in multiple sclerosis? J Clin Exp Neuropsychol. 2004; 26:550-62.

50. Rao SM, et al. Processing speed test: validation of a self-administered, iPad ${ }^{\oplus}$ based tool for screening cognitive dysfunction in a clinic setting. Mult Scler J. 2017;23:1929-37.

51. Patel VP, Shen L, Rose J, Feinstein A. Taking the tester out of the SDMT: A proof of concept fully automated approach to assessing processing speed in people with MS. Mult. Scler. J. 2018;1352458518792772. https://doi.org/10. $1177 / 1352458518792772$.

\section{Publisher's Note}

Springer Nature remains neutral with regard to jurisdictional claims in published maps and institutional affiliations.

Ready to submit your research? Choose BMC and benefit from:

- fast, convenient online submission

- thorough peer review by experienced researchers in your field

- rapid publication on acceptance

- support for research data, including large and complex data types

- gold Open Access which fosters wider collaboration and increased citations

- maximum visibility for your research: over $100 \mathrm{M}$ website views per year

At BMC, research is always in progress.

Learn more biomedcentral.com/submissions 